\title{
Announcements $\cdot$ Mitteilungen
}

\author{
La Conduite Automatique de l'Extrusion et de l'Injection des Plastiques \\ SYMPOSIUM CEMP-EAHP \\ Paris 9-10 Décembre 1980 \\ Hôtel Nikko - 61, quai de Grenelle - Paris 15è
}

Pour permettre un dialogue entre tous les partenaires intéressés, universitaires et industriels rattachés à des disciplines très différentes, et satisfaire ainsi un besoin réciproque de connaissances, tant sur le plan de la technique que du simple langage, le Centre d'étude des matières plastiques organise, en collaboration avec l'Ecole d'application des hauts polymères, un symposium les 9 et 10 décembre 1980 à Paris - Hôtel Nikko - sur la Conduite automatique de l'injection et de l'extrusion des plastiques, deux procédés de transformation qui intéressent en tonnage plus de $60 \%$ des polymères transformés.

La première journée portera sur les bases théoriques et physiques de la conduite automatique, avec 6 conférences:

- Analyse fonctionnelle des procédés de transformation des plastiques pour la conduite automatique.

- Théorie de la conduite automatique optimale des processus industriels. Notions de systèmes asservis multivariables, d'identification, de commande hiérarchisée et d'optimisation statique et dynamique.

- Les organes de conduite par microprocesseur des procédés de transformation des plastiques. Description, fonctions, coûts du matériel et du logiciel, formation du personnel, développement.

- Modélisation de l'écoulement des polymères en extrusion.

- Modélisation du cycle d'injection pour la conduite automatique des presses.

- Modélisation de l'écoulement des polymères dans les moules d'injection appliquée à la conception des moules.
La deuxième journée sera résenrée à l'état des réalisations industrielles (description, fiabilité, rentabilité) avec les conférences suivantes:

- Commande centralisée pour la gestion et la surveillance d'un atelier d'injection.

- De la logique cablée à la logique programmable pour une conduite automatique sintelligente« du procédé d'injection.

- Les développements dans la conduite automatique des presses d'injection en fonction des problèmes périphériques d'ordres techniques (connaissance du procédé) et socio-économiques (formation du personnel, relations clients-fournisseurs ...).

- Conduite automatique d'une chaîne d'extrusion-soufflage de films.

- Conduite automatique d'une ligne d'extrusion de gaines de cables ou de profilés.

- Automatisation d'une chaine d'extrusion-soufflage de flacons bi-orientés.

Ces journées s'adressent aux chercheurs automaticiens et plasticiens, aux constructeurs d'automatismes, d'outillages et de machines de transformation, aux transformateurs et aux producteurs de matières plastiques.

Les conférences seront présentées et présidées par des personnalités appartenant aux différents secteurs intéressés.

Renseignements - Réservations - Programme: CEMP-65, rue de Prony - 75854 Paris Cedex 17 Tél. 763.12.59 - Télex: Inplast 641636 F

Für die Schriftleitung verantwortlich: Prof. Dr.H. Giesekus, Abteilung Chemietechnik der Universität Dortmund, Postfach 500500, D-4600 Dortmund 50

Anzeigenverwaltung und Verlag: Dr. Dietrich Steinkopff Verlag, Postfach 111008, D-6100 Darmstadt 11 Gesamtherstellung: Druckerei Dr. A. Krebs, Hemsbach/Bergstr. und Bad Homburg v.d. H. 